\title{
Self-Efficacy and Psychological Status among Hospitalized Burned Patients
}

\author{
Amal Bakr Abo El-Ata, Abeer Elsayed Berma \\ Lecturer of Medical Surgical Nursing, Faculty of Nursing, Port Said University; \\ Lecturer of Psychiatric Nursing and Mental Health, Faculty of Nursing, Port Said \\ University.
}

\begin{abstract}
Background: Burn injury is often a devastating event with long-term physical and psychosocial effects. It can result in multiple psychological sequelae.Aim: The aim of the current study was to investigate the relationship between self-efficacy and psychological status among hospitalized burned patients.Subject and Methods :the research design A descriptive correlation design was adopted in the current study. Setting: The study was carried out in burn units at Port-Said general hospital, PortSaid, Egypt. Convenient sample of 92 adult burned patients were recruited to participate in this study. Two tools are used for data collection were: the Depression Anxiety Stress Scale (DASS) and General Self-Efficacy Scale (GSE),Result: The results of the current study revealed that Psychological impairment was found to be sever and more than half of studied patients at the anxiety, depression and stress. SelfEfficacy was low in about half of burned patients. There were negative correlation between Self-Efficacy and depression and stress but positive correlation with anxiety.Conclusion The study was concluded that there is a significant relation between stress as well as depression and anatomical site of burn, especially in the upper limb. As well as there was a significant relation found between General SelfEfficacy and patient's age. Recommendations: The importance of early psychological intervention to reduce the complications to recognize and treat pre-existing impairments to reduce the complications of long-term remedy.
\end{abstract}

Key words: Hospitalized burned patients - Psychological status- Self-efficacy. 


\section{INTRODUCTION}

Comparatively little attention has been given to the impact of burns on patients' health status after their return to normal life. Burn wounds are severely stressful events that can have a significant impact on the mental health of patients. Burn leads to number of complications as, infections being the most common. In order of frequency, potential complications include: pneumonia, cellulitis, urinary tract infections and respiratory failure. (Herndon 2006). Risk factors for infection include: burns of more than $30 \%$ TBSA, full-thickness burns, extremes of age (young or old), or burns involving the legs or perineum. (Young, et al., 2008).Pneumonia occurs particularly commonly in those with inhalation injuries. Anemia secondary to full thickness burns of greater than 10\% TBSA is common (Granger, 2009). Electrical burns may lead to compartment syndrome due to muscle breakdown. Blood clotting in the veins of the legs is estimated to occur in 6 to $25 \%$ of people (Brunicardi, 2010). The hyper-metabolic state that may persist for years after a major burn can result in a decrease in bone density and a loss of muscle mass (Rojas et al., 2012) Keloids may form subsequent to a burn, particularly in those who are young and dark skinned. Following a burn, person may have significant psychological trauma and experience post-traumatic stress disorder (Michael, 2009).

Burn injuries are an important public health issue in the East Mediterranean Region being one of the leading causes of injury morbidity and mortality(Othman and Kendrick, 2010).. In Bangladesh, Colombia, Egypt and Pakistan, 17\% of children with burns have a temporary disability and $18 \%$ have a permanent disability (WHO, 2014). Intentional burns are also a common cause and occur at high rates in young women, secondary to domestic violence and self-harm (Peck, 2011). Burn survivors experience a series of traumatic assaults to the body and mind which present extraordinary challenges to psychological resilience. Contrary to what might be expected, empirical data regarding the long-term sequelae of burn injury indicate that many burn survivors do achieve a satisfying quality of life and that most are judged to be well-adjusted individuals. However, thirty percent of any given sample of adult burn survivors consistently demonstrate moderate to severe psychological and/or social difficulties. Psychological factors play a significant role in pain and anxiety discomfort imply to a patient and a patient's family that the medical staff consider discomfort a valid issue that will be treated (Sheridan et al, 2000).

Psychological distress occurs in most survivors of severe burn injuries. While each individual experiences psychological distress differently, people with burn injuries often report. While in the hospital, survivors may find they have a lot of time to focus on their burn injury. Many people report having psychological distress several days or a few weeks after they were injured. For most, periods of distress become less frequent and less upsetting after a couple of weeks to a couple of months (Mason et al, 2010). However, about one third of people with major burn injuries continue to feel very 
distressed for up to 2 years. If these problems continue for more than a month or two, you should seek treatment (Smith et al, 2008).

The most fundamental psychological insult of trauma is helplessness in the face of an event which is psychologically and physically overwhelming. In burn trauma this is complicated by the repetitive nature of procedural trauma and the reactivation of the trauma response. Psychological recovery from burn trauma requires that a sense of safety and power in one's life be restored. Burn injury is often, if not always accompanied by losses. Losses vary depending on the individual situation and extent of the burn injury. Grief is a natural, innate, healing process which occurs in response to loss. This process is time limited and has as its function emotional healing and a return to life activities. Grief does not damage self worth, self esteem, or self image. The predominant feelings of grief are sadness, anxiety, longing for that which has been lost, and oftentimes anger about the loss. Anger tends to be unfocused (Bronson, 2014).

Self-efficacy is defined as a person's judgment of their capabilities to organize and execute the courses of action required to attain predetermined types of performances. It is concerned not with the skills one has, but rather with the estimation of what one can attain with the skills one currently possesses (Bandura, 1986). In other term Selfefficacy is a positive emotion and persons (as a general rule) seek out opportunities to display "self-efficacious behaviour" (Judge et al., 2002).

Self-efficacy is a powerful aspect of the self. It is possibly more dynamic than the other aspects as self esteem and self concept because it, more so than others, determines action or inaction. Self-efficacy also determines how much effort people put into dealing with challenges as well as their resilience in the face of adversity. Additionally it "influences the amount of stress and anxiety individuals experience while engaged in a task (Dormann et al., 2006).

Persons with a strong sense of efficacy are highly motivated to take on challenges, seeing them as tasks to be mastered. Failure is not a deterrent, rather commitment and determination increases with each setback. These individuals are generally a pleasure to be around due to their positive and energetic outlook. Individuals who suffer from low self-efficacy tend to have a negative attitude towards problems which arise, often viewing them "tougher than they really are" (Pajares, 2001). These persons frequently experience bouts of depression and anxiety as a result of their lack of skills, and often avoid "interpersonal interactions"

\section{AIM OF STUDY:}

The aim of this study was to investigate the relationship between self-efficacy and psychological status among hospitalized burned patients. 


\section{SUBJECT AND METHODS:}

\section{Technical Design:}

Research design: A descriptive correlation design was adopted in the current study.

Setting: The study was conducted at burn unit at Port Said general Hospital.

\section{Sampling design}

Target Population: The population of this study was hospitalized burned patients admitted to burn unit in Port Said general hospital from January to September 2012. Sample size: 92 patients and fulfilling the following criteria: Both sexes, Adult patients (20 to 60) year, Moderate degree of burn: extent from 15\% - 25\% TBSA for second degree, and less than $10 \%$ for third degree, Patient after emergent phase(48 hours from burn injury)

Exclusion criteria: patient with inhalation injury, and patient with associated trauma

\section{Tools for data Collection:-}

Two tools were used in the study for data collection.

Tool (1): Depression Anxiety Stress Scale (DASS): it consists of three parts

-Part (1): Socio demographic characteristics of patients such as age, sex, marital status, level of education and number of children

-Part (2): Clinical data such as co-existed medical diagnosis and burn parameter (cause of burn, circumstances of burn, sits and place of burn, degree of burn and extent of burn (total body surface area).

-Part (3): Depression Anxiety Stress Scale (DASS). The standardized version of DASS developed by Lovibond and Lovibond (1995) was used. It is one of the recent scales screening normal adolescents and adults. The DASS is a 42 items self-report inventory that yields negative aspect of emotions, namely stress, anxiety and depression. Each of the three parts of the DASS scale contains 14 items.

Tool (2): General Self Efficacy scale: English version of it developed by Schwarzerand Jerusalem (1995) in four point scale $1=$ not at all true $2=$ hardly true $3=$ moderately true $4=$ exactly true

\section{Scoring system}

\section{DASS Scale:}

Patients were asked to use 4-point severity/frequency scale to rate the extent to which they had experienced each state over the past week. Scores for depression, anxiety and stress were calculated by summing the scores for the relevant items, and categorized as

\begin{tabular}{|l|l|l|l|}
\hline Stress & Anxiety & Depression & \\
$0-14$ & $0-7$ & $0-9$ & Normal \\
\hline $15-18$ & $8-9$ & $10-13$ & Mild \\
\hline $19-25$ & $10-14$ & $14-20$ & Moderate \\
\hline $26-33$ & $15-19$ & $21-27$ & Severe \\
$34-$ & $20-$ & $28-$ & Extremely Severe \\
\hline
\end{tabular}

General Self Efficacy scale: 
Scale point scored as $، 1=$ Not at all true, $\quad 2=$ Hardly true, $\quad 3=$ Moderately, true $4=$ Exactly true, Total converted to percent as Low (>60\%), Fair $(60 \%-<75 \%)$, High $(75 \% \leq)$

\section{(II) Operational Design:}

\section{Content Validity:}

DASS scale and General Self-Efficacy scale translated into Arabic language by the researchers to facilitate its use by patients. A jury of 7 experts in the field of nursing and medicine reviewed the translation of the scales; some changes have been made to ensure that Arabic translation gives the same meaning as the English statements.

\section{Pilot Study:}

The pilot study was carried out on $10 \%$ of the study subjects, which represented 10 patients selected to test the tools before starting the data collection phase, and they were excluded from the entire sample of the research work. Purposes of the pilot study were to test the applicability of the study tools, and it served to estimate the time needed to complete the tools.

\section{Method of Data Collection:}

The actual study was conducted during the academic year 2013-2014 from 20 October 2013 to 31 March 2014, one day each week. The researcher met the patients in their hospital unit and explained to them the purpose of the study, how to complete the study tools and took their oral consent. Patients were also informed about their right to refuse to participate in the study and that their answers will not be taken against them, it would be used for the purpose of the study only. The patients filled in the tools using self-report technique that allow them to fill in their answers by themselves. The researcher was available for any questions from the patients. Each patient took about 60-90 minutes in filling the tools. The researcher was available for helping illiterate patients to fill their tools.

\section{Ethical Considerations:}

1- Hospital permission was taken to carry out the study.

2- Oral consent was obtained from the study subjects before participation in the study.

3- The aim of the study was explained to each participant to be familiar with the importance of his participation and assured them about the confidentiality of the obtained information.

4- Patients were informed about their right to refuse to participate in the study or withdraw at any time.

\section{Field work:}

The 92 patients were randomly selected from the previous setting according to the previous criteria. A written formal consent was obtained from each selected patient for participation in the study after explaining the aim of the study, establishing rapport and trusting relationship with the studied patients. The form of the study tools were 
then explained to the patients and the patients were reassured that all information will be confidential and used only for the purpose of the study and they were individually interviewed for keeping their privacy. The tools were collected by the researcher using the interview method on an individual basis. Each interview lasted for about from 60-90 minutes according to patient attention, concentration and willing to cooperate or talk A number of 2 patients were interviewed per day. The patient's clinical data were checked from their clinical charts to be implemented in the tools. Data were collected over a period of four months starting from first of June and ending August 2015. Two days per week (Tuesday, Thursday), from 20 October 2013 to 31 March 2014, and one day per week to collect the required data. Data were then categorized by the researcher, checked and revised.

\section{Administrative Design}

Before the study carried out: An official letter was addressed from the Dean of the Faculty of Nursing to the director of the identified study setting, requesting his cooperation and permission to conduct the study after explaining the aim of the study, an oral consent was taken from the patients, after explaining the purpose and the importance of the research study. Patients assured about the confidentiality of the information gathered and that it will be used only for the purpose of the study.

\section{Statistical Design}

Data were collected, organized, tabulated and statistically analyzed with SPSS 14.0 software computer statistical. Data were presented numbers and percentages, for qualitative data and means and standard deviation for Quantitative data. Qualitative categorical variables were compared using chi-square test. Whenever the expected values in one or more of the cells in a $2 \times 2$ tables was less than 5, Fisher exact test was used instead. In larger than $2 \times 2$ cross-tables, no test could be applied whenever the expected value in $10 \%$ or more of the cells was less than 5 . Person correlation analysis was used for assessment of the inter-relationships among quantative variables. Statistical significance was considered at $\mathrm{P}$-value $<0.05$

\section{Limitations of the study:}

In line with most researchers, this study had few limitations

-Although the sample size was appropriate for the study, it was relatively small. Thus, it is difficult to generalize the obtained data.

-Dealing with subjects was relatively difficult. Some of them refused to participate in the study even after ensuring the confidentiality.

\section{RESULTS:}

Table (1): illustrates score of Depression, Anxiety and Stress among the studied burned patients. It indicates that the most prominent increase is related to the anxiety which reached $94.6 \%$. Meanwhile, the scores in the depression tend to decline $68.5 \%$. Nonetheless, the more than half of the patients (59.8\%) are stressful. 
Table (2)::shows the Score of General Self-Efficacy among studied burned patients. It revealed that nearly half of the patients $(48.9 \%)$ has low Score of General SelfEfficacy while $(22.8 \%)$ of sample reported high score.

Table (3): shows the Correlation between General Self-Efficacy scores and DASS score. It demonstrated that there are negative correlation between self-efficacy score with depression and stress score while correlation between self-efficacy scales is positive in addition there is no statistically significant correlation between General Self-Efficacy scores and DASS score. ( $\mathrm{P}$ value $=0.922,0.874$ and 0.573 respectively).

Table (4): illustrates score of General Self-Efficacy Scale among studied burned patients according to their socio demographic characteristics. It indicates that statistically significant relation is found between General Self-Efficacy and patients' age, and having children ( $\mathrm{P}$-value $=0.007,0.01$ respectively). There is no statistically significant relation between general Self-Efficacy and the rest of socio demographic characteristics.

Table (5): shows Depression Score among studied burned patients according to their Socio demographic characteristics. It found that there is no statistically significant relation between Depression and the Socio demographic characteristics of burned patients. $(\mathrm{P}$ value $=0.561,0.134,0.976,0.959$ and 0.16 respectively $)$.

Table (6): displays Stress among studied burned patients according to their socio demographic characteristics. It reported that there is no statistically significant relation between stress and the Socio demographic characteristics of burned patients ( $\mathrm{P}$ value $=0.199,0.419,0.018,0.309,0.376$ and 0.024 respectively $)$.

Table (7): shows the relation between scores of General Self-Efficacy and burn characteristics of the studied burned patients . It presents no statistically significant relation is found between General Self-Efficacy and burn characteristics $(\mathrm{P}$ value $=$ $0.874,0.066$ and 0.956 respectively).

Table (8): shows Depression among studied burned patients according to their burn characteristics. It is found that there is a statistically significant relation between depression and the anatomical site of burn in the upper limb. (P value $=0.029$ ).

Table (9): demonstrates stress among the studied burned patients according to their burn characteristics. It displays there is a statistically significant relation between stress and the anatomical site of burn in the upper limb ( $\mathrm{P}$ value $=0.046$ ). 
Table (1): Score of Depression, Anxiety and Stress among the studied burned patients $(n=92)$.

\begin{tabular}{|l|l|c|c|c|c|c|c|c|c|c|}
\hline \multirow{2}{*}{ DASS scale } & \multicolumn{9}{|l|}{ Studied burned patients } \\
\cline { 2 - 15 } & \multicolumn{2}{|c|}{ Normal } & \multicolumn{2}{|c|}{ Mild } & \multicolumn{2}{|c|}{ Moderate } & \multicolumn{2}{c|}{ Severe } & \multicolumn{2}{c|}{ Very severe } \\
\cline { 2 - 14 } & No. & $\%$ & No. & $\%$ & No. & $\%$ & No. & $\%$ & No. & $\%$ \\
\hline Depression scale & 0 & 0.0 & 0 & 0.0 & 12 & 13.0 & 17 & 18.5 & 63 & 68.5 \\
\hline Anxiety scale & 0 & 0.0 & 0 & 0.0 & 1 & 1.1 & 4 & 4.3 & 87 & 94.6 \\
\hline Stress scale & 1 & 1.1 & 2 & 2.2 & 16 & 17.4 & 18 & 19.6 & 55 & 59.8 \\
\hline
\end{tabular}

Table (2): Score general Self-Efficacy among studied burned patients $(n=92)$.

\begin{tabular}{|c|c|c|c|c|c|c|}
\hline \multirow[b]{4}{*}{ General Self-Efficacy } & \multicolumn{6}{|c|}{ Studied burned patients } \\
\hline & \multicolumn{2}{|c|}{ Low $(<60 \%)$} & \multicolumn{2}{|c|}{ Fair $(60 \%-<75 \%)$} & \multicolumn{2}{|c|}{ High $(75 \% \leq)$} \\
\hline & No. & $\%$ & No. & $\%$ & No. & $\%$ \\
\hline & 45 & 48.9 & 26 & 28.3 & 21 & 22.8 \\
\hline
\end{tabular}

Table (3): Correlation between scores of General Self-Efficacy and DASS among the studied burned patients $(n=92)$.

\begin{tabular}{|l|l|l|}
\hline \multirow{2}{*}{ DASS score } & \multicolumn{2}{|l|}{ General Self-Efficacy Scale } \\
\cline { 2 - 3 } & $\mathrm{R}$ & $\mathrm{P}$ \\
\hline Depression score & -0.01 & 0.922 \\
\hline Anxiety score & 0.017 & 0.874 \\
\hline Stress score & -0.059 & 0.573 \\
\hline
\end{tabular}

r: Pearson correlation coefficient 
Table (4): Score of General Self-Efficacy Scale among studied burned patients according to their socio demographic characteristics $(n=92)$.

\begin{tabular}{|c|c|c|c|c|c|c|c|}
\hline \multirow{3}{*}{$\begin{array}{l}\text { Socio demographic } \\
\text { characteristics }\end{array}$} & \multicolumn{6}{|c|}{$\begin{array}{l}\text { General Self-Efficacy Scale for Studied } \\
\text { burned patients }(n=92)\end{array}$} & \multirow{3}{*}{$\begin{array}{l}\text { Significanc } \\
\mathrm{e}\end{array}$} \\
\hline & \multicolumn{2}{|c|}{$\begin{array}{l}\text { Low }(<60 \%) \\
{[n=45]}\end{array}$} & \multicolumn{2}{|c|}{$\begin{array}{l}\text { Fair }(60 \%- \\
<75 \%) \\
{[n=26]}\end{array}$} & \multicolumn{2}{|c|}{$\begin{array}{l}\text { High } \\
(75 \% \leq) \\
{[n=21]}\end{array}$} & \\
\hline & No. & $\%$ & No. & $\%$ & No. & $\%$ & \\
\hline \multicolumn{7}{|l|}{ Age (years) } & \multirow{5}{*}{$\begin{array}{l}\mathrm{X}^{2}=17.564 \\
{ }^{\mathrm{MC}} \mathrm{P}=0.007 \\
*\end{array}$} \\
\hline $20-$ & 20 & 44.4 & 8 & 30.8 & 0 & 0.0 & \\
\hline $30-$ & 9 & 20.0 & 12 & 46.2 & 11 & 52.4 & \\
\hline $40-$ & 11 & 24.4 & 4 & 15.4 & 8 & 38.1 & \\
\hline $50-<60$ & 5 & 11.1 & 2 & 7.7 & 2 & 9.5 & \\
\hline \multicolumn{7}{|l|}{ Gender } & \multirow{3}{*}{$\begin{array}{l}X^{2}=0.7 \\
P=0.705\end{array}$} \\
\hline Male & 24 & 53.3 & 13 & 50.0 & 13 & 61.9 & \\
\hline Female & 21 & 46.7 & 13 & 50.0 & 8 & 38.1 & \\
\hline \multicolumn{7}{|l|}{ Marital status } & \multirow{4}{*}{$\begin{array}{l}X^{2}=7.332 \\
{ }^{M C} \mathrm{P}=0.114\end{array}$} \\
\hline Single & 21 & 46.7 & 10 & 38.5 & 3 & 14.3 & \\
\hline Married & 19 & 42.2 & 13 & 50.0 & 16 & 76.2 & \\
\hline Divorced/widow & 5 & 11.1 & 3 & 11.5 & 2 & 9.5 & \\
\hline \multicolumn{7}{|l|}{ Educational level } & \multirow{5}{*}{$\begin{array}{l}X^{2}=4.564 \\
{ }^{M C} \mathrm{P}=0.619\end{array}$} \\
\hline Illiterate & 9 & 20.0 & 4 & 15.4 & 5 & 23.8 & \\
\hline Basic education & 15 & 33.3 & 10 & 38.5 & 5 & 23.8 & \\
\hline Secondary education & 13 & 28.9 & 4 & 15.4 & 4 & 19.0 & \\
\hline University or higher & 8 & 17.8 & 8 & 30.8 & 7 & 33.3 & \\
\hline \multicolumn{7}{|l|}{ Occupation } & \multirow{6}{*}{$\begin{array}{l}\mathrm{X}^{2}=11.158 \\
{ }_{\mathrm{MC}} \mathrm{P}=0.192\end{array}$} \\
\hline Professional & 13 & 28.9 & 5 & 19.2 & 3 & 14.3 & \\
\hline Office & 4 & 8.9 & 5 & 19.2 & 6 & 28.6 & \\
\hline Manual & 5 & 11.1 & 3 & 11.5 & 6 & 28.6 & \\
\hline Housewife & 9 & 20.0 & 6 & 23.1 & 4 & 19.0 & \\
\hline Not working & 14 & 31.1 & 7 & 26.9 & 2 & 9.5 & \\
\hline \multicolumn{7}{|l|}{ Having children } & \multirow{3}{*}{$\begin{array}{l}\mathrm{X}^{2}=9.134 \\
{ }^{\mathrm{MC}} \mathrm{P}=0.01 *\end{array}$} \\
\hline None & 26 & 57.8 & 14 & 53.8 & 4 & 19.0 & \\
\hline Yes & 19 & 42.2 & 12 & 46.2 & 17 & 81.0 & \\
\hline
\end{tabular}


Table (5): Depression Score among studied burned patients according to their Socio demographic characteristics $(n=92)$

\begin{tabular}{|c|c|c|c|c|c|}
\hline \multirow{3}{*}{ Personal characteristics } & \multicolumn{4}{|c|}{ Depression Scale } & \multirow{3}{*}{$\begin{array}{l}\text { Significan } \\
\text { ce }\end{array}$} \\
\hline & \multicolumn{2}{|c|}{$\begin{array}{l}\text { Less than } v \text {. } \\
\text { severe } \\
{[n=29]}\end{array}$} & \multicolumn{2}{|c|}{$\begin{array}{l}\text { Very severe } \\
{[n=63]}\end{array}$} & \\
\hline & No. & $\%$ & No. & $\%$ & \\
\hline \multicolumn{5}{|l|}{ Age (years) } & \multirow{5}{*}{$\begin{array}{l}\mathrm{X}^{2}=2.138 \\
{ }^{{ }^{M C}} \mathrm{P}=0.561\end{array}$} \\
\hline $20-$ & 10 & 34.5 & 18 & 28.6 & \\
\hline $30-$ & 12 & 41.4 & 20 & 31.7 & \\
\hline $40-$ & 5 & 17.2 & 18 & 28.6 & \\
\hline $50-<60$ & 2 & 6.9 & 7 & 11.1 & \\
\hline \multicolumn{5}{|l|}{ Gender } & \multirow{3}{*}{$\begin{array}{l}X^{2}=2.129 \\
P=0.144\end{array}$} \\
\hline Male & 19 & 65.5 & 31 & 49.2 & \\
\hline Female & 10 & 34.5 & 32 & 50.8 & \\
\hline \multicolumn{5}{|l|}{ Marital status } & \multirow{4}{*}{$\begin{array}{l}\mathrm{X}^{2}=4.06 \\
{ }^{\mathrm{MC}} \mathrm{P}=0.134\end{array}$} \\
\hline Single & 15 & 51.7 & 19 & 30.2 & \\
\hline Married & 12 & 41.4 & 36 & 57.1 & \\
\hline Divorced/widow & 2 & 6.9 & 8 & 12.7 & \\
\hline \multicolumn{5}{|l|}{ Educational level } & \multirow{5}{*}{$\begin{array}{l}X^{2}=0.207 \\
P=0.976\end{array}$} \\
\hline Illiterate & 5 & 17.2 & 13 & 20.6 & \\
\hline Basic education & 10 & 34.5 & 20 & 31.7 & \\
\hline Secondary education & 7 & 24.1 & 14 & 22.2 & \\
\hline University or higher & 7 & 24.1 & 16 & 25.4 & \\
\hline \multicolumn{5}{|l|}{ Occupation } & \multirow{6}{*}{$\begin{array}{l}X^{2}=0.662 \\
{ }^{M C} P=0.959\end{array}$} \\
\hline Professional & 7 & 24.1 & 14 & 22.2 & \\
\hline Office & 4 & 13.8 & 11 & 17.5 & \\
\hline Manual & 5 & 17.2 & 9 & 14.3 & \\
\hline Housewife & 5 & 17.2 & 14 & 22.2 & \\
\hline Not working & 8 & 27.6 & 15 & 23.8 & \\
\hline \multicolumn{5}{|l|}{ Having children } & \multirow{3}{*}{$\begin{array}{l}X^{2}=1.978 \\
P=0.16\end{array}$} \\
\hline None & 17 & 58.6 & 27 & 42.9 & \\
\hline Yes & 12 & 41.4 & 36 & 57.1 & \\
\hline
\end{tabular}

$\mathrm{X}^{2}$ : Chi-Square test

${ }^{\mathrm{MC}}$ P: Monte Carlo corrected P-value 
Table (6): Stress among studied burned patients according to their socio demographic characteristics $(n=92)$.

\begin{tabular}{|c|c|c|c|c|c|}
\hline \multirow{3}{*}{$\begin{array}{l}\text { Socio demographic } \\
\text { characteristics }\end{array}$} & \multicolumn{4}{|c|}{ Stress Scale } & \multirow{3}{*}{$\begin{array}{l}\text { Significan } \\
\text { ce }\end{array}$} \\
\hline & \multicolumn{2}{|c|}{$\begin{array}{l}\text { Less than } v \text {. } \\
\text { severe } \\
{[n=37]}\end{array}$} & \multicolumn{2}{|c|}{$\begin{array}{l}\text { Very severe } \\
{[n=55]}\end{array}$} & \\
\hline & No. & $\%$ & No. & $\%$ & \\
\hline \multicolumn{5}{|l|}{ Age (years) } & \multirow{5}{*}{$\begin{array}{l}\mathrm{X}^{2}=4.762 \\
{ }^{\mathrm{MC}} \mathrm{P}=0.199\end{array}$} \\
\hline $20-$ & 13 & 35.1 & 15 & 27.3 & \\
\hline $30-$ & 14 & 37.8 & 18 & 32.7 & \\
\hline $40-$ & 5 & 13.5 & 18 & 32.7 & \\
\hline $50-<60$ & 5 & 13.5 & 4 & 7.3 & \\
\hline \multicolumn{5}{|l|}{ Gender } & \multirow{3}{*}{$\begin{array}{l}X^{2}=0.652 \\
P=0.419\end{array}$} \\
\hline Male & 22 & 59.5 & 28 & 50.9 & \\
\hline Female & 15 & 40.5 & 27 & 49.1 & \\
\hline \multicolumn{5}{|l|}{ Marital status } & \multirow{4}{*}{$\begin{array}{l}\mathrm{X}^{2}=8.201 \\
{ }^{{ }^{2}} \mathrm{P}=0.018\end{array}$} \\
\hline Single & 20 & 54.1 & 14 & 25.5 & \\
\hline Married & 15 & 40.5 & 33 & 60.0 & \\
\hline Divorced/widow & 2 & 5.4 & 8 & 14.5 & \\
\hline \multicolumn{5}{|l|}{ Educational level } & \multirow{5}{*}{$\begin{array}{l}X^{2}=3.596 \\
P=0.309\end{array}$} \\
\hline Illiterate & 5 & 13.5 & 13 & 23.6 & \\
\hline Basic education & 10 & 27.0 & 20 & 36.4 & \\
\hline Secondary education & 10 & 27.0 & 11 & 20.0 & \\
\hline University or higher & 12 & 32.4 & 11 & 20.0 & \\
\hline \multicolumn{5}{|l|}{ Occupation } & \multirow{6}{*}{$\begin{array}{l}X^{2}=4.228 \\
P=0.376\end{array}$} \\
\hline Professional & 8 & 21.6 & 13 & 23.6 & \\
\hline Office & 6 & 16.2 & 9 & 16.4 & \\
\hline Manual & 5 & 13.5 & 9 & 16.4 & \\
\hline Housewife & 5 & 13.5 & 14 & 25.5 & \\
\hline Not working & 13 & 35.1 & 10 & 18.2 & \\
\hline \multicolumn{5}{|l|}{ Having children } & \multirow{3}{*}{$\begin{array}{l}X^{2}=5.098 \\
P=0.024\end{array}$} \\
\hline None & 23 & 62.2 & 21 & 38.2 & \\
\hline Yes & 14 & 37.8 & 34 & 61.8 & \\
\hline
\end{tabular}

$\mathbf{X}^{2}$ : Chi-Square test $\quad{ }^{M C} \mathbf{P}$ : Monte Carlo corrected P-value 
Table (7): Self-Efficacy among the studied burned patients according to their burn characteristics $(n=92)$.

\begin{tabular}{|c|c|c|c|c|c|c|c|c|}
\hline \multirow{3}{*}{ Burn characteristics } & \multicolumn{7}{|c|}{$\begin{array}{l}\text { General Self-Efficacy for Studied burned } \\
\text { patients }(n=92)\end{array}$} & \multirow{3}{*}{ Significance } \\
\hline & \multicolumn{2}{|c|}{$\begin{array}{l}\text { Low }(\% 60>) \\
\ln =45\end{array}$} & \multicolumn{2}{|c|}{\begin{tabular}{|l} 
Fair $>-\% 60)$ \\
$(\% 75$ \\
]n=26[
\end{tabular}} & \multicolumn{3}{|c|}{$\begin{array}{l}\operatorname{High}(\geq \% 75) \\
\ln =21[\end{array}$} & \\
\hline & No. & $\%$ & No. & $\%$ & No. & $\%$ & & \\
\hline \multicolumn{8}{|l|}{ Cause of burn } & \multirow{4}{*}{$\begin{array}{l}\mathrm{X}^{2}=1.227 \\
{ }^{\mathrm{MC}} \mathrm{P}=0.874\end{array}$} \\
\hline Flame & 24 & 53.3 & 12 & 46.2 & 9 & & & \\
\hline Hot liquids & 11 & 24.4 & 9 & 34.6 & 7 & & & \\
\hline Hot tools & 10 & 22.2 & 5 & 19.2 & 5 & & & \\
\hline \multicolumn{8}{|c|}{ Circumstances of burn } & \multirow{4}{*}{$\begin{array}{l}X^{2}=5.43 \\
{ }^{M C} \mathrm{P}=0.066\end{array}$} \\
\hline Accident & 37 & & 82.2 & 26 & 100.0 & 19 & 90.5 & \\
\hline Criminal/suicide & 8 & & 17.8 & 0 & 0.0 & 2 & 9.5 & \\
\hline \multicolumn{8}{|c|}{ Anatomical site of burn } & \\
\hline Face and Neck & \multicolumn{2}{|l|}{32} & 71.1 & 17 & 65.4 & 13 & 61.9 & $\begin{array}{l}X^{2}=0.619 \\
P=0.734\end{array}$ \\
\hline $\begin{array}{l}\text { Chest and Abdomen } \\
\text { )trunk( }\end{array}$ & \multicolumn{2}{|l|}{37} & 82.2 & 20 & 76.9 & 17 & 81.0 & $\begin{array}{l}\mathrm{X}^{2}=0.299 \\
\mathrm{P}=0.861\end{array}$ \\
\hline Upper limb & \multicolumn{2}{|l|}{25} & 55.6 & 14 & 53.8 & 9 & 42.9 & $\begin{array}{l}X^{2}=0.966 \\
P=0.617\end{array}$ \\
\hline Lower limb & \multicolumn{2}{|l|}{18} & 40.0 & 6 & 23.1 & 6 & 28.6 & $\begin{array}{l}X^{2}=2.349 \\
P=0.309\end{array}$ \\
\hline Genital area & \multicolumn{2}{|l|}{11} & 24.4 & 6 & 23.1 & 6 & 28.6 & $\begin{array}{l}X^{2}=0.202 \\
P=0.904\end{array}$ \\
\hline Place & & & & & & & & \\
\hline Home & 25 & & 55.6 & 12 & 46.2 & 12 & 57.1 & $X^{2}=0.824$ \\
\hline Workplace & 17 & & 37.8 & 12 & 46.2 & 8 & 38.1 & ${ }^{\mathrm{MC}} \mathrm{P}=0.956$ \\
\hline Street & 3 & & 6.7 & 2 & 7.7 & 1 & 4.8 & \\
\hline
\end{tabular}


Table (8): Depression among studied burned patients according to their burn characteristics $(n=92)$.

\begin{tabular}{|c|c|c|c|c|c|}
\hline \multirow{3}{*}{ burn characteristics } & \multicolumn{4}{|c|}{ Depression $) n=92($} & Significance \\
\hline & \multicolumn{2}{|c|}{$\begin{array}{l}\text { Less than } v \text {. } \\
\text { severe } \\
\text { ]n=29[ }\end{array}$} & \multicolumn{2}{|c|}{$\begin{array}{l}\text { Very severe } \\
] \mathrm{n}=63[\end{array}$} & \\
\hline & No. & $\%$ & No. & $\%$ & \\
\hline \multicolumn{6}{|l|}{ Burn characteristics } \\
\hline \multicolumn{5}{|l|}{ Cause of burn } & \multirow{4}{*}{$\begin{array}{l}\mathrm{X}^{2}=0.16 \\
{ }^{\mathrm{MC}} \mathrm{P}=0.923\end{array}$} \\
\hline Flame & 14 & 48.3 & 31 & 49.2 & \\
\hline Hot liquids & 8 & 27.6 & 19 & 30.2 & \\
\hline Hot tools & 7 & 24.1 & 13 & 20.6 & \\
\hline \multicolumn{5}{|l|}{ Circumstances of burn } & \multirow[b]{3}{*}{${ }^{\mathrm{FE}} \mathrm{P}=0.162$} \\
\hline Accident & 28 & 96.6 & 54 & 85.7 & \\
\hline Criminal/suicide & 1 & 3.4 & 9 & 14.3 & \\
\hline \multicolumn{5}{|l|}{ Anatomical site of burn } & \\
\hline Face and Neck & 19 & 65.5 & 43 & 68.3 & $\mathrm{X}^{2}=0.068 \quad \mathrm{P}=0.795$ \\
\hline Trunk (Chest and Abdomen) & 21 & 72.4 & 53 & 84.1 & $\mathrm{X}^{2}=1.731 \quad \mathrm{P}=0.188$ \\
\hline Upper limb & 20 & 69.0 & 28 & 44.4 & $\begin{array}{l}X^{2}=4.785 \\
P=0.029 *\end{array}$ \\
\hline Lower limb & 11 & 37.9 & 19 & 30.2 & $\mathrm{X}^{2}=0.546 \quad \mathrm{P}=0.46$ \\
\hline Genital area & 6 & 20.7 & 17 & 27.0 & $\mathrm{X}^{2}=0.42 \quad \mathrm{P}=0.517$ \\
\hline \multicolumn{5}{|l|}{ Place } & \multirow{3}{*}{$\begin{array}{l}X^{2}=2.755 \\
P=0.28\end{array}$} \\
\hline Home & 12 & 41.4 & 37 & 58.7 & \\
\hline $\begin{array}{l}\text { Workplace } \\
\text { Street }\end{array}$ & $\begin{array}{l}14 \\
3\end{array}$ & $\begin{array}{l}48.3 \\
10.3\end{array}$ & $\begin{array}{l}23 \\
3\end{array}$ & $\begin{array}{l}36.5 \\
4.8\end{array}$ & \\
\hline
\end{tabular}

$\mathrm{X}:{ }^{\mathbf{2}}$ Chi-Square test $\quad{ }^{\mathrm{MC}} \mathrm{P}$ : Monte Carlo corrected P-value ${ }^{\mathrm{FE}} \mathrm{P}$ : Fisher's

Exact test $\quad *$ significant at $\mathbf{P 0 . 0 5} \geq$ 
Table (9): Stress among the studied burned patients according to their burn characteristics $(n=92)$.

\begin{tabular}{|c|c|c|c|c|c|}
\hline \multirow{3}{*}{$\begin{array}{l}\text { Medical history and burn } \\
\text { characteristics }\end{array}$} & \multicolumn{4}{|c|}{ Stress Scale $) n=92($} & Significance \\
\hline & \multicolumn{2}{|c|}{$\begin{array}{l}\text { Less than } \mathbf{v} \text {. } \\
\text { severe } \\
\text { ]n=37[ }\end{array}$} & \multicolumn{2}{|c|}{$\begin{array}{l}\text { Very severe } \\
\text { ]n=55[ }\end{array}$} & \\
\hline & No. & $\%$ & No. & $\%$ & \\
\hline \multicolumn{6}{|l|}{ Burn characteristics } \\
\hline \multicolumn{5}{|l|}{ Cause of burn } & \multirow{4}{*}{$\begin{array}{l}X^{2}=1.78 \\
P=0.409\end{array}$} \\
\hline Flame & 15 & 40.5 & 30 & 54.5 & \\
\hline Hot liquids & 13 & 35.1 & 14 & 25.5 & \\
\hline Hot tools & 9 & 24.3 & 11 & 20.0 & \\
\hline \multicolumn{5}{|l|}{ Circumstances of burn } & \multirow{3}{*}{${ }^{\mathbf{F E}} \mathrm{P}=0.306$} \\
\hline Accident & 35 & 94.6 & 47 & 85.5 & \\
\hline Criminal/suicide & 2 & 5.4 & 8 & 14.5 & \\
\hline \multicolumn{5}{|l|}{ Anatomical site of burn } & \\
\hline Face and Neck & 22 & 59.5 & 40 & 72.7 & $\mathrm{X}^{2}=1.772 \quad \mathrm{P}=0.183$ \\
\hline Trunk (Chest and Abdomen ) & 27 & 73.0 & 47 & 85.5 & $\mathrm{X}^{2}=2.19 \quad \mathrm{P}=0.139$ \\
\hline Upper limb & 24 & 64.9 & 24 & 43.6 & $\begin{array}{l}X^{2}=3.995 \\
P=0.046^{*}\end{array}$ \\
\hline $\begin{array}{l}\text { Lower limb } \\
\text { Genital area }\end{array}$ & $\begin{array}{l}14 \\
8\end{array}$ & $\begin{array}{l}37.8 \\
21.6\end{array}$ & $\begin{array}{l}16 \\
15\end{array}$ & $\begin{array}{l}29.1 \\
27.3\end{array}$ & $\begin{array}{l}X^{2}=0.77 \quad P=0.38 \\
X^{2}=0.377 \quad P=0.539\end{array}$ \\
\hline \multicolumn{5}{|l|}{ Place } & \multirow{4}{*}{$\begin{array}{l}\mathrm{X}^{2}=2.508 \\
{ }^{\mathrm{MC}} \mathrm{P}=0.281\end{array}$} \\
\hline Home & 17 & 45.9 & 32 & 58.2 & \\
\hline Workplace & 16 & 43.2 & 21 & 38.2 & \\
\hline Street & 4 & 10.8 & 2 & 3.6 & \\
\hline
\end{tabular}

$\mathrm{X}:{ }^{2}$ Chi-Square test ${ }^{\mathrm{MC}} \mathrm{P}$ : Monte Carlo corrected P-value ${ }^{\mathrm{FE}} \mathrm{P}$ : Fisher's Exact test *significant at P0.05 $\geq$

\section{DISCUSSION:}

Major burn injuries can be extremely upsetting and distressing for the survivor, as well as for family members, and friends. Common causes of distress include 'worries about the future 'thinking about the event itself - both in sustaining the injury and/or witnessing others who were also seriously injured or died (Mason et al., 2010).

The present study aimed to investigate the relationship between self-efficacy and psychological status among hospitalized burned patients. The majority of the present study sample were indicates the most prominent increase was related to the anxiety. This is probably due to multiple demographic, clinical and environmental factors. Most of the patients were see themselves as ineffective and helpless. The fact that 
they may look and act "different" often excludes them from the ordinary social interactions that provide opportunities to develop one's social self.

These results are in agreement with Wallis et al., (2006) who mentioned that, the results showed significantly greater values of emotional distress among patients when compared with norms of the general population. As well as higher levels of general psychopathology, particularly prevalent were anxiety, depression, and posttraumatic symptoms.

This result is congruent with Tedstone \& Tarrier (1997) and Hulbert-Williams et al., (2008) the greatest impact of the injury was on levels of anxiety and response to trauma-related stress anxiety, depression, and post-traumatic stress disorder are the most commonly observed psychological effects.

Klinge et al., (2009) added that, Physical functioning plays a large role in the cause of depression, anxiety and post-traumatic stress disorder are the most commonly observed psychological effects. Physical functioning plays a large role in the cause of depression which does not always reduced over time many burn survivors displayed signs of depression over 1 year post injury. Anxiety is often related to body image and grievance over ones new image or avoidance of reflection. Many burn victims display 2 or more signs of stress. It is said that those most likely to experience stress are those who were found to have had pre-burn psychosocial problems.

However, Dyster-Aas et al., (2008) indicated that, burn patients have a higher incidence of pre-existing psychopathology than the general population. Pre-existing psychiatric disorders, alcohol, substance abuse and depression play a statistically significant causal role in these injuries.In these regard,

Furthermore, Pfitzer (2014) mentioned that reduced cognitive processes related to disregard for self-protection lead to. reported anxiety is frequently depression, anxiety in burn patients may occur due to psychosocial matters, such as grieving over the loss of their previous appearance or troubled by reactions of others Hulbert-Williams et al ,(2008) suggests that sufferers of major burns experience higher levels of distress when compared with those who have minor burns. In contrast Tebble et al., (2004) claims that injuries no mater what size may have a psychological impact on a patient according to literature Smith (2000) These three psychological disorders are commonly experienced in burn centers 'nurses and staff should be aware of these implications monitor for any signs so that it can possibly be managed before fully developing causing severe stress and trauma.

Moreover, Sveen et al., (2011) have found that Psychological problems are common after burns, and symptoms of posttraumatic stress disorder (PTSD) are some of the most prevalent. There are subgroups among patients with burns that have different patterns of PTSD symptom development. These findings may have implications for clinical practice, such as the timing of assessment and the management of patients 
who present with these symptoms. Additionally, Roh et al., (2012) have stressed that depression is one of the most common psychological problems arising after a burn.

Current results indicated that incidence of symptoms of depression is relatively high, and depressed burn patients report worse burn scar or sensation and lower levels of burn-specific health. Furthermore, Dyster-Aas et al, (2008) who stated that two-third of burn survivors exhibit a history of lifetime psychiatric disorders. Those with a psychiatric history had a higher risk of post burn psychiatric problems. Van Loey $\&$ Van Son (2003) and Patterson et al., (2003) added that, burn patients scored higher on psychological distress, anxiety, depression, and loss of behavioral and emotional control. These results reflect other studies in the literature, depicted that burned patients were pre-morbidly more psychologically vulnerable than the general population, a factor that likely contributes to many of them sustaining their injuries.

As well as, Menzies (2000) indicated that the bio-psychosocial impact of a burn injury on the individual hospitalized for severe burn wounds begins at the moment of injury and extends throughout that person's life. Medical and emotional problems do not exist in clinical isolation but instead interact to confound and complicate treatment outcomes, accentuating the importance of providing optimal treatment of patients' medical illnesses without neglecting their mental distress.

This result was contradicted by Ptacek et al., (2002) stated that the results indicated that few patients rated their depression as severe at any point in time. Depression scores decreased significantly across the hospitalization period and were correlated with burn size, trait anxiety, and well-being. Depression ratings after discharge were significantly related to depression scores obtained at the end of the inpatient phase of the study. Although most patients did not report experiencing severe levels of depression, the stability of scores across time suggests the usefulness of early screening procedures.

The present study noted that the lowest percentage general self-efficacy. This latter finding may be due to the fact that, with burn injuries often report, the most fundamental psychological insult of trauma is helplessness in the face of an event which psychologically and physically overwhelming. This goes with Pajares (2001) Individuals who suffer from low self-efficacy tend to have a negative attitude towards problems which arise, often viewing them "tougher than they really are. These persons frequently experience bouts of depression and anxiety as a result of their lack of skills, and often avoid "interpersonal interactions. On the contrary, Wallis et al., (2006) found that, burn patients reported high levels of resources such as general optimism, self-efficacy, and perceived social support.

Regarding patient's age, the findings of current study revealed that there was significant relation between General Self-Efficacy and patient's age .This result was in line with Wasiak et al (2014) patients of specific demographics, such as female patients and older patients, and patients with a higher percentage of full thickness 
surface area burns are of greater risk for poorer physical and psychological outcomes and may benefit from additional monitoring and rehabilitation

.The present study findings also indicated that there was significant relation between stress as well as depression and anatomical site of burn, especially in the upper limb. Needless to say that the presence of burn in the upper limb can be a source of social, psychological as well as physical problems in patients life. This finding is congruent with Lil. (2002) who have reported that, anxiety and depression in patients with severe burn or facial burn occurred earlier and severer and the patients worried about the possibility to resume their work, the maintenance of family relation, and the ability to join the society, Severe psychological disorders might delay the time of patients' ability to resume their previous work.

In contradiction with the current results, Nicolosi, et al., (2013) stated that Burn location did not affect depression. Thus, it is of great importance to assess the self efficacy and psychological status among burn patients in order to identify and minimize as much as possible the factors of dissatisfaction, provide appropriate management that improve patients' self efficacy and psychological status and offer them opportunities for personal social fulfilling in their lives.

\section{CONCLUSION:}

Based on study findings, it can be concluded that: The physical aspects affected the burned patient, as well as the psychological aspects. The physical and the psychological aspects were related to the anxiety, however symptoms of depression was relatively high, and depressed burned patients reported worse burn scar or sensation. The present study depicted that there was a significant relation between stress, depression and anatomical site of burn, especially in the upper limb. There was a significant relation found between General Self-Efficacy and patient's age.

\section{RECOMMENDATIONS:}

Based on the results of the present study, the following recommendations were suggested:

1- The importance of early psychological intervention to reduce the complications to recognize and treat pre-existing impairments to reduce the complications of longterm remedy.

2- In-service training program should be applied for burned patients to teach them problem-solving skills, assertiveness skills, anger management and new coping skills to enable them to manage stress, anxiety and depression in a constructive way.

3- Screening for these disorders and treatment should be carried out as part of a holistic routine in all burn units because catching such problems early may head off longer-term difficulties. 


\section{REFERENCES:}

Bandura, A. (1986): Social foundations of thought and action: A social cognitive theory. Englewood Cliffs, NJ: Prentice-Hall Inc.

Bronson M( 2014 ): (Psychological and Emotional Impact of a Burn InjuryThis article reprint provided by: The Phoenix Society, Inc. ${ }^{\circledR} \bullet 1835 \mathrm{R}$ W Berends Dr. SW • Grand Rapids, MI 49519-4955 • 800.888.BURN • ttp://www.phoenix-society.org

Brunicardi, C (2010): "Chapter 8: Burns". Schwartz's principles of surgery (9th Ed.). New York: McGraw-Hill, Medical Pub. Division, ISBN 978 -0 - 07-154769 -7.

Conversano C, Lensi E, Testi C, Gremigni P (2010): The burn patient: factors associated with post-traumatic stress disorder and direhctions for intervention. Riv Psichiatr, Jul-Aug;45(4):221-33.

Dahl O, Wickman M, Wengström Y (2012): Adapting to life after burn injury-reflections on care. J Burn Care Res, Sep-Oct; 33(5):595-605.

Dormann, C.; Fay, D.; Zapf, D.; Frese, M. (2006): "A state-trait analysis of job satisfaction: On the effect of core self-evaluations". Applied Psychology: An International Review 55 (1): 27-51. doi:10.1111/j.1464-0597.2006.00227.x

Dyster-Aas J, Willebrand M, Wikehult B, Gerdin B, Ekselius L (2008):Major depression and posttraumatic stress disorder symptoms following severe burn injury in relation to lifetime psychiatric morbidity. Journal of Trauma Injury Infection and Critical Care.1356-1349،(5)64

Granger, $J$ (2009). "An Evidence-Based Approach to Pediatric Burns" Pediatric Emergency Medicine Practice 6 (1).

Herndon D (2006.): "Chapter 3: Epidemiological, Demographic, and Outcome Characteristics of Burn Injury". Total burn care (4th ed.). Edinburgh: Saunders. p. 23. ISBN 978 -1- 4377-2786-9.

\section{Hulbert-Williams N.J., Hulbert-Williams S.L ‘.Mcilroy $D$ \& .BuntingB)(2008)}

Anxiety in recovery from severe burn injury: An experimental comparison. Psychology, Health \& Medicine.167-162,(2)3.

Judge, Timothy A.; Erez, Amir; Bono, Joyce E.; Thoresen, Carl J. (1 January 2002): "Are measures of self-esteem, neuroticism, locus of control, and generalized self-efficacy indicators of a common core construct?". Journal of Personality and Social Psychology 83 (3): 693-710.

Klinge K et al) .2009) :Psychological adjustments made by postburn injury patients: an integrative literature review. Journal of Advanced Nursing 2292-2274(11)65. 
Li L (2002): Correlative analysis of the psychological disorder with ability to resume work in burn patients. Oct; 18(5):305-7. Zhonghua Shao Shang Za Zhi. Article in Chinese.

Lovibond SH and Lovibond PF. Manual for Depression Anxiety Stress Scales. $2^{\text {nd }}$ ed. Sydney: Psychology Foundation; 1995

Mason, S. T ‘.Fauerbach JA ‘Haythornthwaite J(2010)OAssessment of Acute Pain, Pain Relief and Pain Satisfaction. Chapter 41 .in D.C. Turk and R .Melzack) Eds . Handbook of Pain Assessment: Third Edition, Guilford Press: New York, NY.

Mason, S. T., Corry, N., Gould, N., Amoyal, N., Gabriel, V., Wiechman Askay, S., Holavanahalli, R., Banks, S., Arceneaux, L. L., Fauerbach, J. A. (2010): Growth Trajectories of Distress in Burn Patients. Journal of Burn Care Research 31(1): 64-72.

Menzies V(2000):Depression and burn wounds. Arch Psychiatr Nurs. ;14(4):199-206.

Nicolosi T, de Carvalho F, Sabatés L (2013): A quantitative, cross-sectional study of depression and self-esteem in teenage and young adult burn victims in rehabilitation. Ostomy wound Mangement, 59(9):22-9.

Nicolosi T J, Fernandes de Carvalho V, Llonch Sabatés A (2013): A quantitative, cross-sectional study of depression and self-esteem in teenage and young adult burn victims in rehabilitation. " Ostomy wound Mangement Sep;59(9):22-9.

Othman N. and Kendrick D.(2010) : Epidemiology of burn injuries in the East Mediterranean Region: a systematic review. BMC Public Health 2010, 10:83 http://www.biomedcentral.com/1471-2458/10/83

Pajares $\boldsymbol{F}$ (2010): Self-efficacy Lecture [Online .[Retrieved October28 'from the World Wide Web: http//:www.emory.edu/EDUCATION/mfp/efftalk.html

Patterson DR ${ }^{I}$, Finch CP, Wiechman SA, Bonsack R, Gibran N, Heimbach D (2003):Premorbid mental health status of adult burn patients: comparison with a normative sample. J Burn Care Rehabil. Sep-Oct;24(5):347-50.

Peck, MD (November 2011). "Epidemiology of burns throughout the world. Part I: Distribution and risk factors". Burns : journal of the International Society for Burn Injuries 37 (7): 1087-100. doi:10.1016/j.burns.2011.06.005. PMID 21802856.

Pfitzer B, Katona LJ, Lee SJ, O'Donnell M, Cleland H, Wasiak J, Ellen S (2014): Three Years After Black Saturday: Long-Term Psychosocial Adjustment of Burns Patients As a Result of a Major Bushfire, journal burn care research Dec 10.

Ptacek JT, Patterson DR, Heimbach DM(2002): Inpatient depression in persons with burns. J Burn Care Rehabil. Jan-Feb; 23(1):1-9. 
Roh YS, Chung HS, Kwon B, Kim G (2012): Association between depression, patient scar assessment and burn-specific health in hospitalized burn patients. Burns Journal, 38(4):506-12.

Rojas Y, Finnerty CC, Radhakrishnan RS, Herndon DN(December2012): "Burns: an update on current pharmacotherapy". Expert Opin Pharmacother 13 (17): 2485-94. doi:10.1517/14656566.2012.738195. PMC 3576016. PMID 23121414.

Roberts, edited by Michael C. (2009). Handbook of pediatric psychology. (4th ed.). New York: Guilford. p. 421.ISBN 978-1-60918-175-8.

Schwarzer, R., \& Jerusalem, M. (1995). Generalized Self-Efficacy scale. In J. Weinman, S. Wright, \& M. Johnston, Measures in health psychology: A user's portfolio. Causal and control beliefs (pp. 35-37). Windsor, UK: NFER-NELSON.

Shakespeare $V$ (1998): Effect of small burn injury on physical, social and psychological health at 3-4 months after discharge BURSN Accepted: May 18; volume 24, Issue 8, Pages 739-744.

Sheridan, R. L., Hinson, M. I., Liang, M. H., Nackel, A. F., Schoenfeld, D.

A., Ryan, C. M., Mulligan, J. L., and Tompkins, R. G.(2000): Long-Term Outcome of Children Surviving Massive Burns. Jama-Journal of the American Medical Association 1-5-2000; 283(1):69-73.

Smith MT, Klick B, Kozachik S, Edwards RR, Holavanahalli R, Wiechman S, Blakeney P, Lezotte D, Fauerbach JA. (2008): Sleep onset insomnia symptoms during hospitalization for major burn injury predict chronic pain. Pain. Sep 15;138(3):497-506.

Smith H).(2000) :(Challenging Disfigurement. Community Practitione.637(6)73.

Sveen J, Ekselius L, Gerdin B, Willebrand M (2011): A prospective longitudinal study of posttraumatic stress disorder symptom trajectories after burn injury. $\mathbf{J}$ Trauma. Dec;71(6):1808-15.

Tebble N.J., Thomas D.W \& .Price P) .(2004):Anxiety and self-consciousness in patients with minor facial lacerations. Journal of Advanced Nursing 426-417,(4)47.

Tedstone JE $\boldsymbol{I}^{\boldsymbol{l}}$, Tarrier N.(1997): An investigation of the prevalence of psychological morbidity in burn-injured patients. Burns. Nov-Dec;23(7-8):550-4.

There was a significant correlation between individuals' perception of the severity of the injury and the degree of distress experienced $(\mathrm{p}<0.05)$.

Van Loey NE, \& Van Son MJ (2003): Psychopathology and psychological problems in patients with burn scars: epidemiology and management. Am J Clin Dermatol. 4(4):245-72. 
Wallis H, Renneberg B, Ripper S, Germann G, Wind G, Jester A.(2006): Emotional distress and psychosocial resources in patients recovering from severe burn injury. Journal of burn care research, 27(5):734-41).

Wiechman SA (2011): Psychosocial recovery, pain, and itch after burn injuries. Phys Med Rehabil Clin N Am May;22(2):327-45.

Wasiak J, Lee SJ, Paul E, Mahar P, Pfitzer B, Spinks A, Cleland H, Gabbe B. (2014) :Jun;40(4):568-74. Predictors of health status and health-related quality of life 12 months after severe burn, journal burn care research.

World Health Organization (2014): Burns, Fact sheet $\mathrm{N}^{\circ} 365$, available at http://www.who.int/mediacentre/factsheets/fs365/en/, accessed in 1/2016

Young, Christopher King, Fred M. Henretig, ed. (2008): Textbook of pediatric emergency procedures. $2^{\text {nd }}$ ed., Philadelphia: Wolters Kluwer Health/Lippincott Williams \& Wilkins. p. 1077.ISBN 978-0-7817-5386-9. 


\title{
الكفاءة الذاتية و الحالة النفسية لاى مرضى الحروق اثناء وجودهم بالمستشفى
}

\author{
دأمل بكر أبو العطا، د. عبير السيد برمة

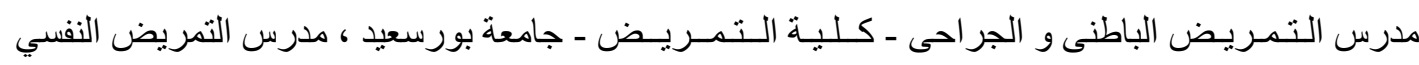 \\ و الصحة العقلية - كلية التمريض - جامعة بورسعيد

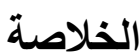

الاصابة بـالحروق غالبا مـا تكون حدث مدمر فى حياة الثخص جسمانباو نفسيا على المدى البعيد. لذلك

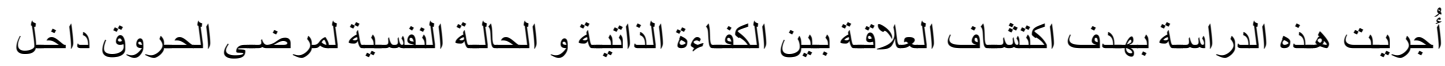

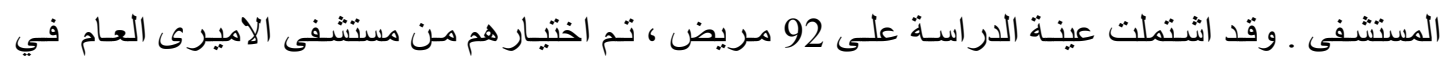

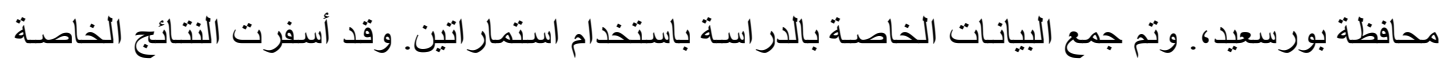

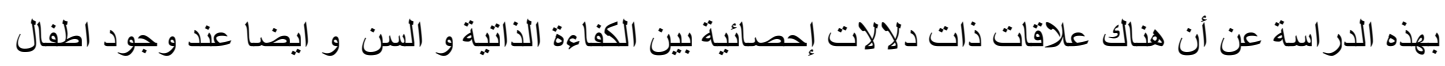
وكذلك وجدت علاقات ذات دلالات إحصائية بين الضغط النفسى و الاكتئاب مع مكان الحرق. وقد أوصت

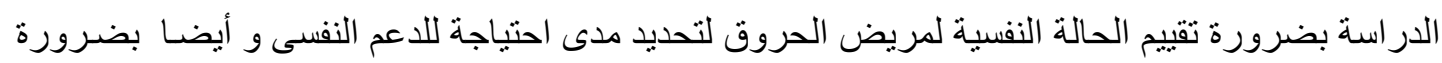

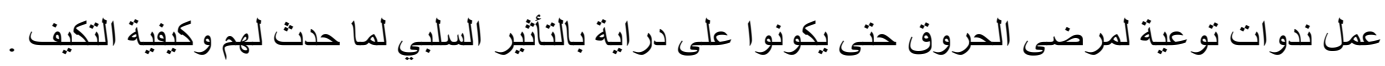

